\title{
Nonfragile Reliable Guaranteed Cost Controller Design for Networked Control System with Variable Sampling Period Sensors
}

\author{
Nan Xie ${ }^{*}$, Bin Xia, Yede Li and Panjing Li \\ School of Computer Science and Technology, Shandong University of Technology, Zibo, 255049, P.R. China
}

\begin{abstract}
In this paper, the problem of nonfragile reliable guaranteed cost control for networked control systems with time-varying sampling period sensors and actuator failures is concerned for a given quadratic cost function. For linear time-invariant controlled plant, under the assumption that the sampling period is time-varying within a certain known bound, the time delay is constant and shorter than the sampling period of the sensors, the system is transformed into a time-varying discrete time system, where the time-varying sampling period parts are transformed into norm bounded uncertainties of the structure parameter. A new linear matrix inequality (LMI)-based approach is proposed to derive a sufficient condition for the existence of nonfragile reliable guaranteed cost controller. Furthermore, the design method of the optimal nonfragile reliable guaranteed cost controller is formulated to minimize the upper bound of the closed-loop system cost. A numerical example is given to show the usefulness and effectiveness of the proposed method.
\end{abstract}

Keywords: Guaranteed cost control, LMI, networked control system (NCS), nonfragile, variable sampling period.

\section{INTRODUCTION}

Recently, networked control systems (NCSs) have been induced into the research of control systems for the reasons of the developing expansion of system physical setups and functionalities. NCSs are a kind of distributed control systems, in which sensors, actuators, and controllers are interconnected by different types of communication networks. Because of its flexibility, low cost, and less wiring, NCSs is increasingly being used in the field of industrial applications. Furthermore, more and more interests have recently been paid to the research of NCSs [1-5].

However, due to the sampling and controlling data are transmitted via the network, network-induced delays and data package dropouts always exist. For NCSs with different kinds of scheduling protocols, the network-induced delays may be in the forms of constant, time-varying, or even random. More recently, the stabilization controller design and stability analysis for NCSs have been investigated by many researchers, and many methods are proposed with the considerations of the effects of network-induced delay and data dropout [6-9]. Results on stability analysis and controller design of nonlinear networked control systems have been obtained in many papers. In [10], based on approximate discrete-time models constructed for a set of nominal (nonzero) sampling intervals and nominal delay, the controller design is propo-sed, while sampling-and-hold effects are taken into account. For a class of nonlinear NCSs, [11] presents a fault-tolerant control framework, decoupled from the system fault, the system is transformed into two subsystems.
The nonlinear observer is designed to estimate the immeasurable state and modeling uncertainty, which are used to construct fault estimation algorithm. For that nonlinear NCS, considering the sampling intervals induced by the network communication, a fault-tolerant control method is proposed

In recent years, there are many papers considering the problem of time-varying sampling period of networked control systems $[12,13]$. For the NCSs, the shorter sampling period results in the better the system performance. However, the possibility of network congestion would be increased by the shorter sampling period. The novel active-varying sampling period method is used by Wang and Yang [13] to eliminate the probability of packet disordering, which greatly simplifies the design and analysis of NCSs, and sufficiently uses the network bandwidth when the network is idle. For the NCSs with stochastic disturbance, [14] gives the conditions of robust stability, and Gao et al. [15] change the stochastic sampling system into a continuous time-delay system, and research the robust $H_{\infty}$ control problem. In [16], the nonstatic observer and state controller are designed, with no disturbances and noises are taken into account, and the application condition of the separation principle is discussed. The dynamic interval principle is used by Liu et al. to design the corresponding controller [17], but the algorithm proposed is complicated and needs a great deal of computations. In [18], for the system with uncertain and time-varying sampling period and time-delay, the stability and control issues of systems are addressed, the sampling period which is uncertain and time-varying and the time delay are transformed into polytopic and additive norm-bounded uncertainties in the discretized system. A Markov chain is modeled for randomly sampled measurement process results from the 
time-varying channel load in [19], an event-driven transmitter is proposed to transmit the control signal, which depends upon the measurement sampling period.

The paper is organized as follows. In section 2, for the given linear time-invariant controlled plant, assume that the time delay is constant and the time delay is shorter than the sampling period, the sampling is time-varying and within a certain known bound, the system is transformed into a timevarying discrete system, where the time-varying sampling period parts are transformed into norm bounded uncertainties of the structure parameters. In section 3, the LMI-based sufficient condition for the existence of nonfragile reliable guaranteed cost controllers is obtained. Furthermore, the optimal reliable guaranteed cost controller which minimizes the upper bound of the closed-loop system cost is formulated. To decrease the amount of LMIs for stability analysis, the reduction algorithms are proposed. In section 4, a numerical simulation result shows the validity of the controller design and the reduction algorithm.

\section{PROBLEM STATEMENT}

Consider the following NCS;

$$
\left\{\begin{array}{l}
\dot{x}(t)=\mathrm{A}_{c} x(t)+\mathrm{B}_{c} u(t) \\
y(t)=\mathrm{C}_{c} x(t)
\end{array}\right.
$$

where $\quad x(t) \in R^{n} \quad$ is the state vector, $u(t)=\left[\begin{array}{llll}u_{1}(t) & u_{2}(t) & \cdots & u_{m}(t)\end{array}\right]^{\mathrm{T}} \in R^{m}$ is the control vector, and $y(t)$ is the output vector.

Denote $\tau_{S C}^{k}$ as the sensor-to-controller network transmission time delay, $\tau_{C A}^{k}$ as the controller-to-actuator time delay, and $\tau_{C}^{k}$ is the actuator computation time delay. Due to the static memoryless state feedback controller is concerned here, the controller could be removed to the actuator end, thus, we denote $\tau_{k}=\tau_{S C}^{k}+\tau_{C A}^{k}+\tau_{C}^{k}=\tau$ as a constant, the system is under the assumptions as following:

(1) The time delay is shorter than a sampling period, that is $\tau<h_{\text {min }}$;

(2) Time varying sampling period is $T_{k}=h_{k}+\tau$, due to $h_{k}$ is time-varying and bounded, so the sampling period of the NCS is time-varying and bounded, thus $T_{k} \in\left[T_{\min }, T_{\max }\right]$;

(3) $h_{0}$ is the nominal value of $h_{k}$, thus, $h_{k}=h_{0}+\Delta_{k}$, the uncertain part of the time-varying sampling period is $\Delta_{k}$;

(4) The actuators and the controllers are event-driven, the sensors are clock-driven, and the sampling instant is $t_{k}$, the sampling period is $T_{k}=t_{k+1}-t_{k}$.
The zero-order-holder $(\mathrm{ZOH})$ at the actuator end ensures the $u\left(t-\tau_{k}\right)$ be the same one when $t \in\left[t_{k}, t_{k}+\tau\right)$ and $t \in\left[t_{k}+\tau, t_{k+1}\right)$, that is:

$u(t)=\left\{\begin{array}{l}u\left(t_{k-1}\right), \quad t_{k} \leq t<t_{k}+\tau \\ u\left(t_{k}\right), \quad t_{k}+\tau \leq t<t_{k+1}\end{array}\right.$

The discretized system according to the sampling period $h_{k}$ is obtained as follows:

$$
\begin{aligned}
& x(k+1)=\mathrm{e}^{A\left(h_{k}+\tau\right)} x(k) \\
& +\int_{k T_{k}}^{k T_{k}+\tau} \mathrm{e}^{A_{C}\left(k T_{k}+T-s\right)} \mathrm{d} s B_{C} u(k-1) \\
& +\int_{k T_{k}+\tau}^{k T_{k}+T_{k}} \mathrm{e}^{A_{C}\left(k T_{k}+T-s\right)} \mathrm{d} s B_{C} u(k) \\
& =\mathrm{e}^{A\left(h_{k}+\tau\right)} x(k)+\mathrm{e}^{A_{C} h_{k}} \int_{0}^{\tau} \mathrm{e}^{A_{C} s} \mathrm{~d} s B_{C} u(k-1) \\
& +\int_{0}^{h_{k}} \mathrm{e}^{A_{C} s} \mathrm{~d} s B_{C} u(k)
\end{aligned}
$$

Denote $H=\left[\begin{array}{cc}A_{c} & B_{c} \\ 0 & 0\end{array}\right]$, where $A_{c}$ and $B_{c}$ are constant matrices of appropriate dimensions, then:

$$
F(T) \triangleq \exp (H T)=\left[\begin{array}{cc}
\exp \left(A_{c} T\right) & \int_{0}^{T} \exp \left(A_{c} s\right) B_{c} \mathrm{~d} s \\
0 & I
\end{array}\right]
$$

Introducing the augmented vector $z(k)=\left[\begin{array}{ll}x^{\mathrm{T}}(k) & u^{\mathrm{T}}(k-1)\end{array}\right]^{\mathrm{T}}$, then (3) can be formed as;

$z(k+1)=\Phi\left(h_{k}\right) z(k)+\Gamma\left(h_{k}\right) u(k)$

$\Phi\left(h_{k}\right)=\left[\begin{array}{cc}\mathrm{e}^{A_{c}\left(h_{k}+\tau\right)} & \mathrm{e}^{A_{c} h_{k}} \int_{0}^{\tau} \mathrm{e}^{A_{c} s} \mathrm{~d} s B_{c} \\ 0 & 0\end{array}\right]$

$=A_{0}+\left[\begin{array}{l}I \\ 0\end{array}\right]\left[\begin{array}{ll}\mathrm{e}^{A_{c} \Delta k}-I & \int_{0}^{\Delta k} \mathrm{e}^{A_{c} s} B_{c} \mathrm{~d} s\end{array}\right] A_{0}$

$=A_{0}+\varphi^{-1}\left[\begin{array}{l}I \\ 0\end{array}\right] \varphi \int_{0}^{\Delta k} \mathrm{e}^{A_{c} s} B_{c} \mathrm{~d} s\left[\begin{array}{ll}A_{c} & B_{c}\end{array}\right] A_{0}=A_{0}+D F_{k} E_{1}$

$\Gamma\left(h_{k}\right)=\int_{0}^{h_{k}} \mathrm{e}^{A_{C} s} \mathrm{~d} s B_{C} u(k)$

$=B_{0}+\left[\begin{array}{l}I \\ 0\end{array}\right]\left[\begin{array}{ll}\mathrm{e}^{A_{c} \Delta k}-I & \int_{0}^{\Delta k} \mathrm{e}^{A_{c} s} B_{c} \mathrm{~d} s\end{array}\right] B_{0}$

$=B_{0}+D F_{k} E_{2}$ 
where;

$$
\begin{aligned}
& A_{0}=\left[\begin{array}{cc}
\mathrm{e}_{c}^{A_{c}\left(h_{0}+\tau\right)} & \mathrm{e}^{A_{c} h_{0}} \int_{0}^{\tau} \mathrm{e}^{A_{c} s} \mathrm{~d} s B_{c} \\
0 & 0
\end{array}\right]=F\left(h_{0}\right)\left[\begin{array}{ll}
I & 0 \\
0 & 0
\end{array}\right] F(\tau), \\
& B_{0}=\left[\begin{array}{c}
\int_{0}^{h_{0}} \mathrm{e}^{A_{c} s} \mathrm{~d} s B_{c} \\
\mathrm{I}
\end{array}\right]=F\left(h_{0}\right)\left[\begin{array}{l}
I \\
0
\end{array}\right], D=\varphi^{-1}\left[\begin{array}{l}
I \\
0
\end{array}\right], \\
& F_{k}=\varphi \int_{0}^{\Delta k} \mathrm{e}^{A_{c} s} \mathrm{~d} s, E_{1}=\left[\begin{array}{ll}
A_{c} & B_{c}
\end{array}\right] A_{0}, \\
& E_{2}=\left[\begin{array}{ll}
A_{c} & B_{c}
\end{array}\right] B_{0},
\end{aligned}
$$

$\varphi$ is some real constant.

Therefore, the discrete system of system (1) as;

$$
\left\{\begin{aligned}
z(k+1) & =\left(A_{0}+D F_{k} E_{1}\right) z(k)+\left(B_{0}+D F_{k} E_{2}\right) u(k) \\
& \triangleq \bar{A} z(k)+\bar{B} u(k) \\
y(k)= & C z(k)
\end{aligned}\right.
$$

where:

$$
\begin{aligned}
& \bar{A}=A_{0}+D F_{k} E_{1}, \\
& \bar{B}=B_{0}+D F_{k} E_{2}, \\
& C=\left[\begin{array}{ll}
C_{c} & 0
\end{array}\right] .
\end{aligned}
$$

To determine $\varphi$, the following lemmas are given.

Lemma 1. For constant matrix $A_{c},\left\|\mathrm{e}^{A_{c} t}\right\| \leq \mathrm{e}^{\eta t}$ exists for all the $t \geq 0$.

Theorem 1. The uncertain item $F_{k}$ is norm bounded, $|\Delta k| \in\left[0, \Delta_{\max }\right]$, when the real constant $\varphi \neq 0$ and;

$$
|\varphi| \leq \begin{cases}\eta /\left(\mathrm{e}^{\eta \Delta_{\max }}-1\right), & \eta \neq 0 \\ 1 / \Delta_{\max }, & \eta=0\end{cases}
$$

is satisfied, $F_{k}^{\mathrm{T}} F_{k} \leq I$ exists, where $\eta=\frac{1}{2} \sigma_{\max }\left(A_{c}+A_{c}^{*}\right)$, $\sigma_{\max }(\bullet)$ is the largest singular value, $A_{c}^{*}$ is the conjugate transpose matrix of $A_{c}$.

Proof. When $\eta \neq 0$, from lemma 1, we can get:

$$
\begin{aligned}
& \| F_{k}|| \leq|\varphi| \int_{0}^{\Delta_{\max }}|| \mathrm{e}^{A t}|\mathrm{~d} t \leq| \varphi\left|\int_{0}^{\Delta_{\max }}\right| \mathrm{e}^{\eta t} \mid \mathrm{d} t \\
& =\mid \varphi\left(\mathrm{e}^{\eta \Delta_{\max }}-1\right) / \eta
\end{aligned}
$$

Obviously, when $|\varphi| \leq \eta /\left(\mathrm{e}^{\eta \Delta_{\max }}-1\right)$, we obtain $F_{k}^{\mathrm{T}} F_{k} \leq I$. When $\eta \neq 0$, the proof is omitted.

Associated with this system is the cost function;

$$
J=\sum_{k=0}^{\infty}\left[z^{\mathrm{T}}(k) Q z(k)+u^{\mathrm{T}}(k) R u(k)\right],
$$

where $Q$ and $R$ are given positive-definite matrices.

For the control input $u_{i}(k), i=1,2, \mathrm{~L}, m$, let $u_{i}^{F}(k)$ denote the signal from the actuator that has failed. The following failure model is adopted in this paper;

$u_{i}^{F}(k)=\alpha_{i} u_{i}(k), \quad i=1,2, \cdots, m$

where;

$0 \leq \widehat{\alpha}_{i} \leq \alpha_{i} \leq \breve{\alpha}_{i}, \quad i=1,2, \cdots, m$

with $\widehat{\alpha}_{i} \leq 1$ and $\breve{\alpha}_{i} \geq 1$.

In the above model of actuator failure, if $\widehat{\alpha}_{i}=\breve{\alpha}_{i}$, then it corresponds to the normal case $u_{i}^{F}(k)=u_{i}(k)$. When $\breve{\alpha}_{i}=0$, it covers the outage case. If $\hat{\alpha}_{i}>0$, it corresponds to the partial failure case, namely, partial degradation of the actuator.

Denote;

$$
\begin{aligned}
& u^{F}(k)=\left[\begin{array}{llll}
u_{1}^{F}(k) & u_{2}^{F}(k) & \cdots & u_{m}^{F}(k)
\end{array}\right]^{\mathrm{T}}, \\
& \breve{\alpha}=\operatorname{diag}\left\{\breve{\alpha}_{1}, \breve{\alpha}_{2}, \cdots, \breve{\alpha}_{m}\right\} \text {, } \\
& \widehat{\alpha}=\operatorname{diag}\left\{\widehat{\alpha}_{1}, \widehat{\alpha}_{2}, \cdots, \widehat{\alpha}_{m}\right\} \text {, } \\
& \alpha=\operatorname{diag}\left\{\alpha_{1}, \alpha_{2}, \cdots, \alpha_{m}\right\} \text {, }
\end{aligned}
$$

$\alpha$ is said to be admissible if $\alpha$ satisfies $\widehat{\alpha} \leq \alpha \leq \breve{\alpha}$.

Define;

$$
\begin{aligned}
& \beta=\operatorname{diag}\left\{\beta_{1}, \beta_{2}, \cdots, \beta_{m}\right\}, \\
& \beta_{0}=\operatorname{diag}\left\{\beta_{10}, \beta_{20}, \cdots, \beta_{m 0}\right\}
\end{aligned}
$$

where:

$$
\beta_{i}=\frac{\widehat{\alpha}_{i}+\breve{\alpha}_{i}}{2}, \quad \beta_{i 0}=\frac{\breve{\alpha}_{i}-\widehat{\alpha}_{i}}{\breve{\alpha}_{i}+\widehat{\alpha}_{i}}, \quad i=1,2, \cdots, m
$$

From (11) and (12), we define;

$\alpha=\left(I+\alpha_{0}\right) \beta$

and;

$$
\left|\alpha_{0}\right| \leq \beta_{0} \leq I
$$


$\alpha_{0}=\operatorname{diag}\left\{\alpha_{01}, \alpha_{02}, \cdots, \alpha_{0 m}\right\}$

$\left|\alpha_{0}\right|=\operatorname{diag}\left\{\left|\alpha_{01}\right|,\left|\alpha_{02}\right|, \cdots,\left|\alpha_{0 m}\right|\right\}$.

The objective of this paper is to develop a procedure to design a memoryless state feedback control law;

$u(k)=(K+\Delta K) z(k)$,

such that for any admissible uncertain $\alpha$, the resulting closed-loop system;

$z(k+1)=(\bar{A}+\bar{B} \alpha(K+\Delta K)) x(k)$

is asymptotically stable and cost function (8) satisfies $J \leq J^{*}$, where $J^{*}$ is some specified constant.

In the controller (16), $K$ is the nominal controller gain, and $\Delta K$ represents the gain perturbations. In this paper, the following two types of perturbations are considered:

(a) $\Delta K$ is of the additive form;

$\Delta K=M_{1} F_{1}(k) N_{1}, \quad F_{1}^{T}(k) F_{1}(k) \leq I$,

where $M_{1}$ and $N_{1}$ are known constant matrices, and $F_{1}(k)$ is the uncertain parameter matrix.

(b) $\Delta K$ is of the multiplicative form;

$\Delta K=M_{2} F_{2}(k) N_{2} K, \quad F_{2}^{T}(k) F_{2}(k) \leq I$,

where $M_{2}$ and $N_{2}$ are known constant matrices, and $F_{2}(k)$ is the uncertain parameter matrix.

Definition 1. A control law $u(k)=(K+\Delta K) z(k)$ is said to be a reliable guaranteed cost control law associated with a cost matrix $P>0$ for system (1) and cost function (8), if the following matrix inequality;

$[\bar{A}+\bar{B} \alpha(K+\Delta K)]^{\mathrm{T}} P[\bar{A}+\bar{B} \alpha(K+\Delta K)]$

$-P+Q+(K+\Delta K)^{\mathrm{T}} \alpha R \alpha(K+\Delta K)<0$

holds for all admissible $\alpha$ and uncertain matrix $F_{k}$ satisfying $F_{k}^{\mathrm{T}} F_{k} \leq I$.

Lemma 2. (Schur complement). Given the constant matrices $\Omega_{1}, \Omega_{2}, \Omega_{3}$ of appropriate dimensions, where $\Omega_{1}=\Omega_{1}^{\mathrm{T}}$ and $\Omega_{2}=\Omega_{2}^{\mathrm{T}}>0$, then $\Omega_{1}+\Omega_{3}^{\mathrm{T}} \Omega_{2}^{-1} \Omega_{3}<0$ if and only if:

$\left[\begin{array}{cc}\Omega_{1} & \Omega_{3}^{\mathrm{T}} \\ \Omega_{3} & -\Omega_{2}\end{array}\right]<0$ or $\left[\begin{array}{cc}-\Omega_{2} & \Omega_{3} \\ \Omega_{3}^{\mathrm{T}} & \Omega_{1}\end{array}\right]<0$.

Lemma 3. [20]. Given matrices $Y, H, E$ of appropriate dimensions and with $Y$ symmetric, then for all $F$ satisfying $F^{\mathrm{T}} F \leq I$ and $Y+H F E+E^{\mathrm{T}} F^{\mathrm{T}} H^{\mathrm{T}}<0$, if and only if there exists $\varepsilon>0$ such that $Y+\varepsilon H H^{\mathrm{T}}+\varepsilon^{-1} E^{\mathrm{T}} E<0$.

\section{MAIN RESULTS}

The following theorem gives the nonfragile reliable guaranteed cost controller for systems (1) and (8).

Theorem 2. For the system (1) and the cost function (8), if there exist matrices $X>0, R_{0}>0, Y$, and scalars $\gamma>0$, $\varepsilon>0$ such that for any admissible $\alpha$, the following matrix inequality holds;

\begin{tabular}{|c|c|c|c|c|c|}
\hline$\gamma D D^{\mathrm{T}}-X$ & $B_{0} R_{0}$ & & $+B_{0} \beta Y$ & $B_{0}$ & \\
\hline$*$ & $-R^{-1}+$ & & $\beta Y$ & 0 & \\
\hline$*$ & $*$ & & $-X$ & 0 & \\
\hline$*$ & $*$ & & $*$ & $-R_{0}^{-1}$ & \\
\hline$*$ & $*$ & & $*$ & $*$ & \\
\hline$*$ & $*$ & & $*$ & $*$ & \\
\hline$*$ & $*$ & & $*$ & $*$ & \\
\hline$*$ & $*$ & & $*$ & $*$ & \\
\hline$*$ & $*$ & & $*$ & $*$ & \\
\hline 0 & 0 & 0 & $B_{0} \beta M_{1}$ & 0 & \\
\hline$\left(E_{2} R_{0}\right)^{\mathrm{T}}$ & 0 & 0 & $\beta M_{1}$ & 0 & \\
\hline$\left(E_{1} X+E_{2} \beta Y\right)^{\mathrm{T}}$ & $X$ & $Y^{\mathrm{T}} \beta \beta_{0}$ & 0 & $\left(N_{1} X\right)^{\mathrm{T}}$ & \\
\hline$E_{2}^{\mathrm{T}}$ & 0 & 0 & 0 & 0 & \\
\hline$-\gamma I$ & 0 & 0 & $E_{2} \beta M_{1}$ & 0 & $<0$ \\
\hline$*$ & $-Q^{-1}$ & 0 & 0 & 0 & \\
\hline$*$ & $*$ & $-R_{0}$ & $M_{1} \beta \beta_{0}$ & 0 & \\
\hline$*$ & $*$ & $*$ & $-\varepsilon^{-1} I$ & 0 & \\
\hline$*$ & $*$ & $*$ & $*$ & $-\varepsilon I$ & \\
\hline
\end{tabular}

where $(*)$ denotes the symmetric element of a matrix, then;

$u(k)=Y X^{-1} z(k)$

is a reliable guaranteed cost controller, the corresponding closed-loop cost function satisfies:

$J \leq z_{0}^{\mathrm{T}} P z_{0}$.

Proof. Define Lyapunov function $V(z)=z^{\mathrm{T}} P z$, denote $\bar{K} \triangleq K+\Delta K$, from Definition 1 , for all the non-zero $z(k)$ and $F_{k}^{\mathrm{T}} F_{k} \leq I$, the derivative of $V(z)$ along any trajectory of the closed-loop system;

$z(k+1)=(\bar{A}+\bar{B} \alpha \bar{K}) z(k)$

satisfies;

$\Delta V(z)=V(z(k+1))-V(z(k))$

$\leq-z(k)^{\mathrm{T}}\left(Q+\bar{K}^{\mathrm{T}} \alpha R \alpha \bar{K}\right) z(k)<0$, 
So the closed-loop system is quadratic stable. Furthermore, from (16), we can obtain:

$z(k)^{\mathrm{T}} Q z(k)+u(k)^{\mathrm{T}} \alpha R \alpha u(k)<-\Delta V(z)$,

By summing both sides of the above inequality from $k=0$ to $k=\infty$, due to $z(\infty)=0$, we get:

$$
\sum_{k=0}^{\infty}\left(z(k)^{\mathrm{T}} Q z(k)+u(k)^{\mathrm{T}} \alpha R \alpha u(k)\right) \leq z_{0}^{\mathrm{T}} P z_{0} .
$$

It is sufficient to show that (20) holds for all the admissible $\alpha$ and $\bar{K}$, by the Schur complement, $P>0, R>0$ and inequality (16) is equivalent to:

$$
\left[\begin{array}{ccc}
-P^{-1} & 0 & \bar{A}+\bar{B} \alpha \bar{K} \\
* & -R^{-1} & \alpha \bar{K} \\
* & * & Q-P
\end{array}\right]<0 .
$$

By (14), (15) and (24), and using the inequality $2 a^{\mathrm{T}} b \leq a^{\mathrm{T}} a+b^{\mathrm{T}} b$ for any diagonal matrix $R_{0}>0$, it follows that:

$$
\begin{aligned}
& {\left[\begin{array}{ccc}
-P^{-1} & 0 & \bar{A}+\bar{B} \alpha \bar{K} \\
* & -R^{-1} & \alpha \bar{K} \\
* & * & Q-P
\end{array}\right]} \\
& =\left[\begin{array}{ccc}
-P^{-1} & 0 & \bar{A}+\bar{B} \beta \bar{K} \\
* & -R^{-1} & \beta \bar{K} \\
* & * & Q-P
\end{array}\right]+\left[\begin{array}{c}
\bar{B} \\
I \\
0
\end{array}\right] \alpha_{0} \\
& \text { - }\left[\begin{array}{lll}
0 & 0 & \beta \bar{K}
\end{array}\right]+\left(\left[\begin{array}{c}
\bar{B} \\
I \\
0
\end{array}\right] \alpha_{0}\left[\begin{array}{lll}
0 & 0 & \beta \bar{K}
\end{array}\right]\right)^{\mathrm{T}} \\
& \leq\left[\begin{array}{cc}
-P^{-1}+\bar{B} R_{0} \bar{B}^{\mathrm{T}} & \bar{B} R_{0} \\
* & -R^{-1}+R_{0} \\
* & *
\end{array}\right. \\
& \left.\begin{array}{c}
\bar{A}+\bar{B} \beta \bar{K} \\
\beta \bar{K} \\
Q-P+\bar{K}^{\mathrm{T}} \beta R_{0}^{-1} \beta_{0}^{2} \beta \bar{K}
\end{array}\right]<0
\end{aligned}
$$

Pre- and post-multiplying the left-hand side matrix in (25) by the matrix $\operatorname{diag}\left\{I, I, P^{-1}\right\}$, respectively, define $X=P^{-1}$, using the Schur complement, and Substitute $\bar{A}=A_{0}+D F_{k} E_{1}$
$\bar{B}=B_{0}+D F_{k} E_{2}$ into the obtained inequality, for $F_{k}^{\mathrm{T}} F_{k} \leq I$, there exists a scalar $\gamma>0$, such that:

$$
\begin{aligned}
& {\left[\begin{array}{cccc}
\gamma D D^{\mathrm{T}}-X & B_{0} R_{0} & A_{0} X+B_{0} \beta \bar{K} X & B_{0} \\
* & -R^{-1}+R_{0} & \beta \bar{K} X & 0 \\
* & * & -X & 0 \\
* & * & * & -R_{0}^{-1} \\
* & * & * & * \\
* & * & * & * \\
* & * & * & *
\end{array}\right.} \\
& \left.\begin{array}{ccc}
0 & 0 & 0 \\
\left(E_{2} R_{0}\right)^{\mathrm{T}} & 0 & 0 \\
\left(E_{1} X+E_{2} \beta \bar{K} X\right)^{\mathrm{T}} & X & (\bar{K} X)^{\mathrm{T}} \beta \beta_{0} \\
E_{2}^{\mathrm{T}} & 0 & 0 \\
-\gamma I & 0 & 0 \\
* & -Q^{-1} & 0 \\
* & * & -R_{0}
\end{array}\right]<0
\end{aligned}
$$

Substituting $\bar{K}=K+\Delta K=K+M_{1} F_{1}(k) N_{1}$ into (26), define $Y=K P^{-1}$, for $F_{1}^{\mathrm{T}}(k) F_{1}(k) \leq I$, there exists a scalar $\varepsilon>0$, using the Schur complement again, (21) is obtained. The proof is complete.

Based on Theorem 2, the design problem of the optimal nonfragile reliable guaranteed cost controller can be formulated by solving a certain optimization problem as following.

Theorem 3. Consider the system (1) with cost function (8), if the following optimization problem

$$
\begin{aligned}
& \min _{\gamma, \varepsilon, \zeta, R_{0}, X, Y} \zeta \\
& \text { s.t. (1) }(21), \\
& \text { (2) }\left[\begin{array}{cc}
-\zeta & z_{0}^{\mathrm{T}} \\
z_{0} & -X
\end{array}\right]<0 .
\end{aligned}
$$

has a solution $\gamma, \varepsilon, \zeta, R_{0}, X, Y$, then $u(k)=Y X^{-1} z(k)$ is the optimal nonfragile reliable guaranteed cost controller.

Proof. It follows from the Schur complement that the constraint condition (2) in the (27) is equivalent to $z_{0}^{\mathrm{T}} X^{-1} z_{0}<\zeta$. From the Theorem 2, we obtain the result of the theorem.

Remark 1. When the controller gain perturbation $\Delta K$ is of the multiplicative form (19), the criterion for the 
nonfragile reliable guaranteed cost control of the systems is identical to the LMI (21), except that $M_{1}, N_{1} X$ are changed as $M_{2}, N_{2} Y$, respectively. The proof is omitted.

\section{SIMULATIONS}

For the NCS

$$
\left\{\begin{array}{l}
\dot{x}(t)=\left[\begin{array}{cc}
0 & 1 \\
0 & -0.1
\end{array}\right] x(t)+\left[\begin{array}{c}
0 \\
0.1
\end{array}\right] u(t) \\
y(t)=\left[\begin{array}{ll}
0 & 1
\end{array}\right] x(t)
\end{array}\right.
$$

We take the time-varying part of the sampling period as $h_{k} \in\left[\begin{array}{ll}0.5 & 1.0\end{array}\right] \mathrm{s}$, time delay $\tau=0.4 \mathrm{~s}, Q=0.5 I_{3 \times 3}$, $R=1$. Because $\eta=0.5525 \neq 0,|\varphi| \leq \eta /\left(e^{\eta \Delta_{\max }}-1\right)$. It is assumed that the input to the system has partial failure as follows: $\widehat{\alpha}=0.75, \breve{\alpha}=1.25$. For the $h_{0}=0.5\left(h_{\max }+h_{\min }\right)$, we choose $\varphi=3.1$, and $M_{1,2}=0.1, N_{1,2}=\left[\begin{array}{c}-0.1 \\ 0.1\end{array}\right]^{T}$.

(1) When the gain perturbations $\Delta K$ is of the additive form (18), the corresponding optimal reliable guaranteed cost controller is:

$$
u(k)=\left[\begin{array}{lll}
-0.7692 & -7.5836 & -0.5491
\end{array}\right] z(k),
$$

the associated upper bound over the closed-loop cost function is $J^{*}=101.6916$.

(2) When the gain perturbations $\Delta K$ is of the multiplicative form (19), the corresponding optimal reliable guaranteed cost controller is:

$$
u(k)=\left[\begin{array}{lll}
-0.9384 & -8.6852 & -0.4627
\end{array}\right] z(k),
$$

the associated upper bound over the closed-loop cost function is $J^{*}=128.4815$.

\section{CONCLUSION}

For the given linear time-invariant controlled system, under the assumption that the time delay is constant and the time delay id shorter than the sampling period, the sampling period is time-varying within a certain known bound, the system is transformed into a time-varying discrete time system. Based on the linear matrix inequality (LMI) approach, the design method of nonfragile reliable guaranteed cost controllers is derived. Furthermore, a problem of convex optimization with LMI constraints is formulated to design the optimal controller which minimizes the upper bound of the closed-loop system cost. In order to decrease the numbers of necessary LMIs, reduction algorithms are proposed for stability analysis.

\section{CONFLICT OF INTEREST}

The authors confirm that this article content has no conflict of interest.

\section{ACKNOWLEDGEMENTS}

This work was supported by the Shandong Provincial Natural Science Foundation, China (ZR2011FQ015).

\section{REFERENCES}

[1] D. Yue, Q.-L. Han, and C. Peng, "State feedback controller design of networked control systems", IEEE Transactions on Circuits and Systems-II: Express Briefs, vol. 51, no. 11, pp. 640-644, 2004.

[2] W. Zhang, M.S. Branicky, and S.M. Phillips, "Stability of networked control systems", IEEE Control System Magazine, vol. 21, pp. 84-99, 2001.

[3] AM. Iqbal, R. Ibrahim, and M. Zulfadhli, "Development and implementation of hybrid controllers for flow control application", Sensors \& Transducers, vol. 17, no. 12, pp. 110-124, 2012.

[4] J. Zhang, P. Guo, M. Lv, and Y. Bo, "Fault detection for networked control systems based on wireless sensor networks", Sensors \& Transducers, vol. 22, pp. 119-125, 2013.

[5] M.-B.-O. Mohammad-Reza, G. Ahmad, and K. Jafar, "Intelligent robust nonlinear controller for MEMS angular rate sensor", Sensors \& Transducers, vol. 141, no. 6, pp. 92-105, 2012.

[6] J. Feng, and S.-Q Wang, "Reliable Fuzzy control for a class of nonlinear networked control systems with time delay", Acta Automatica Sinica, vol. 38, no. 7, pp. 1091-1099, 2012.

[7] S. Wen, Z. Zeng, and T. Huang, "Robust probabilistic sampling $\mathrm{H} \infty$ output tracking control for a class of nonlinear networked systems with multiplicative noises", Journal of the Franklin Institute, vol. 350, pp. 1093-1111, 2013.

[8] Y. Shi, and B. Yu, "Robust mixed $\mathrm{H} 2 / \mathrm{H} \infty$ control of networked control systems with random time delays in both forward and backward communication links", Automatica, vol. 47, no. 4, pp. 754-760, 2011

[9] F. Yang, and Q.-L. Han, "Hळ control for networked systems with multiple packet dropouts", Information Sciences, vol. 252, pp. 106-117, 2013

[10] N. van de Wouw, D. Nešić, and W.P.M.H. Heemels. A discretetime framework for stability analysis of nonlinear networked control systems, Automatica, vol. 48, no. 6, pp. 1144-1153, 2012.

[11] Z. Mao, B. Jiang, and P. Shi, Observer based faulttolerant control for a class of nonlinear networked control systems, Journal of the Franklin Institute, vol. 347, no. 6, pp. 940-956, 2010.

[12] W. Chen, and L. Qiu, "Stabilization of networked control systems with multirate sampling", Automatica, vol. 49, no. 6, pp. 1528-1537, 2013.

[13] Y. Wang, and G. Yang, "Hळ Controller design for networked control systems via active-varying sampling period method", Acta Automation Sinica, vol. 34, no. 7, pp. 814-818, 2008.

[14] R.A. Borges, R.C. Oliveira, and C.T. Abdallah, "Robust $\mathrm{H} \infty$ networked control for systems with uncertain sampling rates", Control Theory \& Applications, vol. 4, no. 1, pp. 50-60, 2010.

[15] H. Gao, J. Wu, and P. Shi, "Robust sampled-data Ho control wi-th stochastic sampling", Automatica, vol. 45, no. 7, pp. 1729-1736, 2009.

[16] L. Hetel, J. Daafouz, and C. Iung, "Analysis and control of LTI and switched systems in digital loops via an event-based modeling", International Journal of Control, vol. 81, no. 7, pp. 1125-1138, 2008.

[17] F. Liu, Y. Yao, and F. He, "Stability analysis of networked con-trol systems with time-varying sampling periods", Journal of Control Theory and Application, vol. 6, no. 1, pp. 22-25, 2008.

[18] M. Izák, D. Görges, and S. Liu, "Stabilization of systems with variable and uncertain sampling period and time delay", Nonlinear Analysis: Hybrid Systems, vol. 4, pp. 291-305, 2010. 
[19] Y. Xu, H. Su, and Y.-J. Pan, "Output feedback stabilization for markov-based nonuniformly sampled-data networked control systems", Systems \& Control Letters, vol. 62, pp. 656-663, 2013.
[20] B.R. Barmish, "Necessary and sufficient conditions for quadratic stability of an uncertain system", Journal of Optimization Theory and Applications, vol. 46, pp. 399-408, 1985.

Received: November 26, 2014

(c) Xie et al.; Licensee Bentham Open.

This is an open access article licensed under the terms of the Creative Commons Attribution Non-Commercial License (http://creativecommons.org/licenses/by-nc/3.0/) which permits unrestricted, non-commercial use, distribution and reproduction in any medium, provided the work is properly cited. 